\title{
Avaliação da espiritualidade/religiosidade e opinião de residentes sobre a participação em um módulo de espiritualidade e integralidade
}

\author{
Evaluation of spirituality/religiosity and opinion of \\ residents of recife about the spirituality and integrality \\ module
}

\begin{abstract}
Arturo de Pádua Walfrido Jordán', Arthur Fernandes da Silva', Daniella Bandim Cruz², Leopoldo Nelson Fernandes Barbosa²
\end{abstract}

IInstituto de Medicina Integral Professor Fernando Figueira, Recife (PE), Brasil.

${ }^{2}$ Faculdade Pernambucana de Saúde, Recife (PE), Brasil.

Recebido: Dez. 29, 2020

Aceito: Maio 03, 2021

\section{COMO CITAR ESTE ARTIGO}

Jordán APW, Silva AF, Cruz DB, Barbosa LNF. Avaliação da espiritualidade/ religiosidade e opinião de residentes sobre a participação em um módulo de espiritua ef Health Education. 2021 Mês-Mês;6(1): 1-17. https://doi.org/10.4322/ijhe.2020.013

\section{CORRESPONDÊNCIA}

Arturo de Pádua Walfrido Jordán

Instituto de Medicina Integral Professor Fernando Figueira

Avenida Mal. Mascarenhas de Morais 4861, Imbiribeira, CEP 51150-000, Recife (PE), Brasi

arturojor@yahoo.com.br

\section{FONTE DE FINANCIAMENTO}

Financiado pelos próprios

autores do trabalho.

\section{CONFLITO DE INTERESSE \\ Os autores declararam não}

haver conflitos de interesse.

estudo foi realizado na Secretaria de Saúde do Recife, Recife (PE), Brasil.

Todos os autores leram e aprovam a versão final submetida ao Interdisciplinary Journal of Health Education (IJHE).

\section{RESUMO}

Objetivo: Apesar da formação em espiritualidade e saúde estar sendo amplamente discutida em todo o mundo, no Brasil desenvolve-se lentamente, principalmente nos cursos de pós-graduação. O presente estudo avaliou a espiritualidade/religiosidade e a opinião de residentes sobre a participação em um módulo transversal de espiritualidade e integralidade. Método: Trata-se de estudo exploratório, descritivo, de corte transversal com abordagem quantitativa com amostra de 55 residentes. Os dados foram obtidos através de um questionário semiestruturado e autoaplicável incluindo as escalas Duke Religion Index (DUREL) e Spirituality Self Rating Scale (SSRS). Os dados foram analisados com auxílio do Software Statistical Package for the Social Sciences (SPSS) na versão 13.0 para Windows. O estudo foi aprovado pelo Comitê de Ética em Pesquisa da Faculdade Pernambucana de Saúde com CAAE: 16559619.2.0000.5569 e parecer de número: 3.495.64. Resultados: Participaram 55 residentes, a maioria do sexo feminino, com idade média de 25,41 anos, brancas e católicas. Sobre o ensino em saúde e espiritualidade, 65,45\% julga ser bastante importante a preparação do residente em espiritualidade. Sobre o módulo de espiritualidade e integralidade, 94,55\% considerou muito bom e todos afirmaram que o conteúdo Ihes trouxe contribuições positivas. Sobre a escala DUREL, 41,82\% frequenta algumas vezes templos ou igrejas, 38,18\% realizava leituras de livros sagrados, preces ou orações diariamente. A escala SSRS que avalia o escore de orientação espiritual, obteve média de 23,43 (DP=4,87). Conclusão: Os residentes demostraram uma religiosidade baixa sugerindo que continua sendo um desafio o desenvolvimento da consciência espiritual durante a formação profissional. A orientação espiritual alta, por sua vez, sugere que os residentes julgam importante questões pertinentes às suas dimensões espirituais, embora com dificuldade em aplicá-las às suas próprias vidas. A participação em um módulo sobre a temática mostrou-se relevante contibuindo de forma positiva para a prática profissional dos residentes.

PALAVRAS-ChaVE: Espiritualidade. Religião. Educação em saúde. Internato e residência.

\section{ABSTRACT}

Objective: Although training in spirituality and health is being widely discussed worldwide, in Brazil it still seems to be slower, especially in graduate courses. The present study evaluated spirituality/religiosity, the opinion of residents about training in spirituality and the Transversal Module of Spirituality and Integrality. Methods: This is an exploratory, descriptive, crosssectional study with a quantitative approach with a sample of 55 residents. The data were obtained through a semi-structured and self-administered questionnaire including the Duke Religion Index (DUREL) and Spirituality Self Rating Scale (SSRS). The data were analyzed with the aid of the Software Statistical Package for the Social Sciences (SPSS) in version 13.0 for Windows. The study was approved by the Research Ethics Committee of Faculdade Pernambucana de Saúde with CAAE: 16559619.2.0000.5569 and opinion number: 3,495.64. Results: Most of the 55 residents declared themselves to be female, with an average age of 25.41 years, white and Catholic. Regarding teaching in health and spirituality, the majority (65.45\%) believes that the resident's preparation in spirituality is quite important. Regarding the spirituality and comprehensiveness module, the majority (94.55\%) considered it very good and all stated that the content brought them contributions. On the DUREL scale, the majority (41.82\%) sometimes attend temples or churches, the majority (38.18\%) read 
sacred books, prayers or prayers daily. The SSRS scale, which assesses the spiritual orientation score, obtained an average of 23.43 (SD = 4.87). Conclusion: Residents showed a low religiosity suggesting that the development of spiritual awareness during professional training remains a challenge. High spiritual guidance, in turn, suggests that residents consider issues relevant to their spiritual dimensions to be important, although with difficulty in applying them to their own lives. Finally, training in spirituality proved to be relevant, contributing positively to the residents' professional practice.

KEYWORDS: Spirituality. Religion. Health education. Internship and residency.

\section{Introdução}

Espiritualidade pode ser entendida como uma busca pessoal pelo entendimento de respostas a questões sobre a vida, seu significado e relações com o sagrado e transcendente, que pode ou não estar relacionada a propostas de uma determinada religião ${ }^{1}$. Da mesma forma, pode ser o aspecto da humanidade que se refere a forma como o indivíduo busca e expressa significado e propósito e a maneira como experimenta sua conexão com o momento, consigo mesmo, com os outros e com a natureza ${ }^{2}$.

Uma pesquisa publicada em 2014 ao buscar na base de dados PUBMED todos os artigos publicados abordando espiritualidade, religião e saúde de 1999 a 2013, mostrou um incremento anual significativo de artigos que tratam do tema, passando de 1.547 publicações em 1999 para 2.750 em 2013. Estas publicações trazem importantes evidências acerca da influência da espiritualidade na saúde ligados a promoção a saúde, prevenção de agravos e assistência à saúde ${ }^{3}$.

Espiritualidade tem relação direta com bem-estar e felicidade, esperança, otimismo e autoestima. Ainda mostra relação inversa com depressão, ansiedade e suicídio. Tem efeitos importantes sobre a saúde cardiovascular na medida em que interfere positivamente nos níveis de colesterol, Hipertensão Arterial Sistêmica (HAS), Acidente Vascular Encefálico (AVE), doenças coronarianas agudas e ainda nos níveis de infecção cardiovascular pós-cirúrgicos ${ }^{4,5}$.

Esse corpo de evidências reforçou a necessidade da abordagem da espiritualidade na prática da saúde e a formação dos futuros profissionais na área. Um estudo brasileiro corrobora com este raciocínio, visto ter demonstrado que os profissionais de saúde não se sentem preparados para esta abordagem com seus pacientes ${ }^{6}$.

Nos Estados Unidos, por exemplo, em 2008, em um total de 150 escolas médicas, 100 ofereciam alguma atividade ligada à espiritualidade em seus cursos, e em 75 destas, a frequência a tais atividades tornou-se parte do programa regular de graduação ${ }^{7}$. No Brasil, em 2010, foram avaliadas 47\% das 180 escolas médicas existentes no país e mostrou-se que apenas 10,4\% destas possuíam cursos dedicados ao assunto ${ }^{8}$.

Em relação ao método de abordagem da Espiritualidade, é sabido que pode ser desenvolvido de várias formas, incluindo palestras, discussões em pequenos grupos, entrevistas padronizadas de pacientes, acompanhamento de capelães, leituras específicas e ainda em módulos transversais ${ }^{9,10}$.

O Módulo Transversal de Espiritualidade e Integralidade foi instituido no ano de 2019 no âmbito das Residências em Saúde da Secretaria de Saúde do Recife (SESAURecife), como oferta a sete programas: Residência em Enfermagem em Atendimento Pré-Hospitalar, Residência em Enfermagem Obstétrica, Residência em Odontologia em Saúde Coletiva, Residência Multiprofissional em Vigilância em Saúde, Residência Multiprofissional na Rede de Atenção Psicossocial, Residência Multiprofissional em Saúde Coletiva e Residência Multiprofissional em Saúde da Família.

O conteúdo teórico-prático foi baseado no relatório National Competencies in Spirituality and Health for Medican Education (NCSMD) elaborado pela Association of American Medical Colleges $(A A M C)$, que traça domínios e competências essenciais a serem desenvolvidas pelos estudantes sobre saúde e espiritualidade, destacando: Conhecimento sobre o assunto; Mecanismos de inserção do tema no sistema de saúde; Incorporação da abordagem da espiritualidade na assistência ao paciente; Incorporação da temática no 
desenvolvimento social e profissional; Estabelecimento de presença compassiva e diálogo com a equipe de saúde, paciente e familiares sobre espiritualidade $\mathrm{e}^{11,12}$.

O módulo é ofertado a todos os residentes do primeiro ano dos programas de Residência em Saúde da SESAU, com carga horária de 32 horas. Os residentes são divididos em 4 grupos que desenvolvem 8 encontros de 4 horas cada. O processo de formação é todo baseado em metodologias ativas de aprendizagem utilizando como ferramentas: Aprendizado Baseado em Equipes (ABE), rodas de diálogo, leitura e discussão de artigos, análise e discussão de vídeos, role-playing, exposição dialogada e Feedback $360^{\circ}$.

Neste sentido, o presente estudo objetivou avaliar a espiritualidade/religiosidade dos residentes, bem como as respectivas opiniões acerca do Módulo Transversal de Espiritualidade e Integralidade e a conseguinte formação em espiritualidade.

\section{Método}

Trata-se de um estudo exploratório, analítico, de corte transversal com abordagem quantitativa realizado no âmbito dos programas de Residência em Saúde da SESAU Recife. O estudo contemplou os residentes do primeiro ano dos 7 programas de Residência em Saúde que participaram do Modulo transversal de Espiritualidade e Integralidade em 2019: Residência em Enfermagem em Atendimento Pré-Hospitalar, Residência em Enfermagem Obstétrica, Residência em Odontologia em Saúde Coletiva, Residência Multiprofissional em Vigilância em Saúde, Residência Multiprofissional na Rede de Atenção Psicossocial, Residência Multiprofissional em Saúde Coletiva e Residência Multiprofissional em Saúde da Família.

Da população de 62 residentes do primeiro ano, a amostra conteve 55 participantes, visto que 7 residentes não estavam presentes nos momentos da aplicação dos instrumentos de coleta, que se deu de maio a dezembro de 2019.

Os dados foram obtidos através de questionário semiestruturado e autoaplicável adaptado do estudo multicêntrico transversal - Spirituality and Brazilian Medical Education (Sbrame) - que envolveu 12 escolas médicas brasileiras, com a participação de 5.950 estudantes entre 2010 e $2011^{13}$, respondido após a assinatura do Termo de Consentimento Livre e Esclarecido (TCLE) e contendo: Dados sociodemográficos e formação acadêmica; Dados acerca da temática "saúde e espiritualidade; Dados acerca do ensino em saúde e espiritualidade; Dados acerca da aplicabilidade clínica em saúde e espiritualidade; Dados acerca do módulo transversal em espiritualidade e integralidade; Dados acerca de concepções de espiritualidade e religiosidade e dados obtidos pela Duke Religion Index (DUREL) e Spirituality Self Rating Scale (SSRS) (Anexo A).

A escala DUREL possui cinco itens que medem três dimensões de religiosidade: religiosidade organizacional (RO), religiosidade não organizacional (RNO) e religiosidade intrínseca (RI), sendo: $\mathrm{RO}$ compreende os comportamentos religiosos que ocorrem no contexto da instituição religiosa como frequência igrejas ou templos, RNO engloba os comportamentos religiosos privados ou informais, como leituras e estudos de livros sagrados e preces ou orações e RI é a busca da religião como um bem maior, habitualmente associado à personalidade e estado mental saudáveis, cujas pontuações estão dispostas em uma escala Likert com 5 (RI) ou 6 opções (RO e RNO) e devem ser analisadas separadamente. As opções de resposta da dimensão de religiosidade intrínseca foram oferecidas em direção crescente, onde o escore mais alto (15) é relacionado à maior religiosidade intrínseca e o escore mais baixo (3) é relacionado à menor religiosidade intrínseca ${ }^{14}$.

A SSRS é um instrumento de autopreenchimento composto por seis itens que avaliam aspectos da espiritualidade do indivíduo. O primeiro item trata sobre a importância de passar tempo com pensamentos espirituais particulares e meditações; o segundo, sobre o esforço para viver de acordo com crenças religiosas; 
o terceiro, sobre a relevância que o indivíduo confere aos pensamentos espirituais que tem sozinho, ou então em reuniões religiosas ou espirituais; o quarto, sobre o interesse na leitura de assuntos relacionados à espiritualidade ou religião; o quinto investiga se a espiritualidade ajuda a manter a estabilidade e o equilíbrio da vida; e finalmente o sexto item, sobre a consideração que se dá à espiritualidade como base para a vida. Os respondentes devem marcar uma entre cinco opções que variam de " 1 = concordo totalmente" a " $5=$ discordo totalmente" (Escala de Likert) e as respostas devem ser dadas de acordo com a percepção do indivíduo no momento do preenchimento das questões. Para sua utilização, é necessário fazer o somatório de pontos, que varia de 6 a 30. Para isso, deve-se, anteriormente, recodificar cada item do instrumento (por exemplo, escore de 5 torna-se 1; 2 torna-se 4; e assim por diante). As respostas recodificadas são somadas para produzir o escore total, e este, por sua vez, representa o nível de orientação espiritual ${ }^{15}$.

Os dados foram digitados em um banco de dados construído no Excel para Windows na versão 2016 e posteriormente analisado por um estatístico com auxílio dos softwares Statistical Package for the Social Sciences (SPSS) na versão 13.0 para Windows. As variáveis qualitativas foram resumidas através de distribuição de frequência absolutas (contagem), frequência relativa (porcentagem) e as variáveis quantitativas resumidas através de média, desvio padrão, valor mínimo e máximo. Para análise de associação entre variáveis qualitativas, utilizou-se o teste de qui quadrado. Para análise de associação entre uma variável qualitativa e uma quantitativa, utilizou-se o teste T de Student. Para análise de uma variável qualitativa com mais de duas categorias e uma quantitativa, utilizou-se o teste F. Considerado para fins estatísticos o valor de $\mathrm{p}<0,05$.

O presente estudo foi aprovado pelo Comitê de Ética em Pesquisa da Faculdade Pernambucana de Saúde sob o CAAE: 16559619.2.0000.5569, parecer de número: 3.495.641 e seguiu todos os preceitos éticos incluídos na resolução 466/12 do CONEP.

\section{Resultados}

Durante o período do estudo, foram entrevistados 55 residentes. Com relação às características sociodemográficas, 41 eram do sexo feminino (74,55\%), a idade média era de 25,41 anos ( $\mathrm{DP}=4,34), 29$ (52,73\%) autodeclararam-se da raça branca e 31 (56,36\%) afirmaram dispor de uma renda mensal de 4 a 7 salários-mínimos. Sobre religião, 19 (34,55\%) afirmaram não ter filiação religiosa, 18 (32,73) eram católicos, $11(20 \%)$ Evangélico/Protestante, 6 (10,91\%) Espíritas e 1 (1,82\%) apontou outra denominação.

Acerca das informações sobre a formação acadêmica, 36 (65,45\%) possuíam a graduação como maior título, seguidos de 16 (29,09\%) com especialização/residência, $2(3,64 \%)$ com mestrado e $1(1,82)$ com doutorado. Em relação ao programa de

Tabela 1. Distribuição da frequência das respostas sobre o programa de residência em curso. Recife, 2020.

\begin{tabular}{lcc}
\multicolumn{1}{c}{ Programa de residência em curso } & N=55 & (\%) \\
Residência em Enfermagem em Atendimento Pré-Hospitalar & 00 & 00,00 \\
\hline Residência em Enfermagem Obstétrica & 09 & 16,36 \\
\hline Residência em Odontologia em Saúde Coletiva & 09 & 16,36 \\
\hline Residência Multiprofissional em Vigilância em Saúde & 03 & 5,45 \\
\hline Residência Multiprofissional na Rede de Atenção Psicossocial & 09 & 16,36 \\
\hline Residência Multiprofissional em Saúde Coletiva & 13 & 23,64 \\
\hline Residência Multiprofissional em Saúde da Família & 12 & 21,82 \\
\hline
\end{tabular}


residência em curso, a maioria dos participantes 13 (23,64\%) estava cursando a residência multiprofissional em saúde coletiva (Tabela 1).

Sobre a opinião dos residentes acerca da interferencia da espiritualidade na saúde das pessoas, $52(94,55 \%)$ acreditam que interfere muito ou extremamente e destes, $51(92,72 \%)$ consideram ser geralmente positiva ou igualmente positiva e negativa. Importante frisar que nenhum residente considerou geralmente negativa ou negativa. Em relação à vontade e motivação em abordar a espiritualidade dos pacientes $42(77,77 \%)$ afirmaram que estiveram motivados frequentemente ou algumas vezes.

Ainda sobre os dados acerca da temática "saúde e espiritualidade", apenas $12(21,82 \%)$ afirmaram considerar-se muito preparados e $43(78,19 \%)$ pouco ou moderadamante preparados para abordar a espiritualidade dos pacientes, 50 (90,91\%) afirmaram tal abordagem ser muito ou muitíssimo pertinente. Entretanto, $34(61,81 \%)$ entrevistados sentem-se desencorajados em abordar a espiritualidade dos pacientes, devido à falta de conhecimento teórico ou treinamento prático e 27 (49,09\%), afirmaram ter medo de impor os próprios pontos de vista religiosos ao paciente.

Acerca dos dados sobre o ensino em saúde e espiritualidade, $46(83,64 \%)$ residentes afirmaram que abordaram raramente ou algumas vezes temas referentes à espiritualidade nos campos de prática nas atividades curriculares ao longo da residência. Da mesma forma, $47(87,04 \%)$ afirmaram que, ao longo da formação acadêmica, os docentes/tutores/preceptores nunca ou raramente abordaram temas referentes à espiritualidade (Tabela 2).

Tabela 2. Distribuição da frequência das respostas sobre a frequência de abordagem de temas referentes à espiritualidade por residentes e docentes/tutores/preceptores nas atividades curriculares ao longo do curso. Recife, 2020.

\begin{tabular}{ccccc}
\multicolumn{5}{c}{ Abordagem de temas referentes à espiritualidade durante o curso } \\
& \multicolumn{2}{c}{ Pelos proprios residentes } & \multicolumn{2}{c}{ Pelos docentes/tutores/preceptores } \\
& $\mathbf{N}=\mathbf{5 5}$ & $\mathbf{( \% )}$ & $\mathbf{N}=\mathbf{5 4}$ & $\mathbf{( \% )}$ \\
\hline Nunca & 5 & 9,09 & 21 & 38,89 \\
\hline Raramente & $\mathbf{1 7}$ & 30,91 & 26 & 48,18 \\
\hline Algumas vezes & 29 & 52,73 & 07 & 12,96 \\
\hline Frequentemente & 04 & 7,27 & 00 & 00,00 \\
\hline Sempre & 00 & 00,00 & 00 & 00,00 \\
\hline
\end{tabular}

Ainda sobre os dados sobre o ensino em saúde e espiritualidade, a maioria, $36(65,45 \%)$, julga ser bastante importante a preparação do residente durante sua formação para abordar espiritualidade com os pacientes e $45(81,82 \%)$ de todos os participantes, acham bastante importante incluir o ensino em saúde e espiritualidade como matriz curricular nos programas de residência.

No que diz respeito ao módulo transversal em espiritualidade e integralidade, a maioria dos residentes, $52(94,55 \%)$ considerou muito bom e os $3(5,45 \%)$ restantes bom. Todos os participantes acreditam que o módulo lhes trouxe contribuições, sendo na prática profissional para $53(96,36 \%)$, na vida pessoal para $54(98,18 \%)$ e na vida em sociedade para $48(87,27 \%)$ e a maioria $51(92,73 \%)$ afirmou sentir-se mais preparado para abordar a espiritualidade na prática clínica como profissional de saúde após a participação no módulo. Sobre o conteúdo do módulo que mais acharam interessante a maioria 44 (80\%) afirmou ter sido Espiritualidade e Finitude, seguido de Espiritualidade e Humanização e Espiritualidade na Prática Clínica ambos com 42 (76,36\%) das respostas, Conceitos básicos sobre espiritualidade, religiosidade e religião com 37 (67,27\%), Psiconeuroimunologia com 23 (41,81\%) e Epigenética com 12 (21,81\%). 
Ainda neste cenário foi identificado que 53 residentes (96,36\%) referiram que frequentemente ou sempre estiveram motivados para participar do módulo de espiritualidade e $2(3,64 \%)$ afirmaram que algumas vezes sentiram-se motivados. A maioria 54 (98,18\%) afirmou que a Secretaria deve oferecer o módulo aos futuros residentes e a totalidade dos residentes opina que o Módulo Transversal em Espiritualidade e Integralidade interessa também a residentes de outros programas, inclusive de outras instituições.

Sobre a escala DUREL, na dimensão de Religiosidade Organizacional (RO), a maioria $23(41,82 \%)$ afirmou que frequentava algumas vezes ao ano templos ou igrejas e 11 (20\%) afirmaram que não frequentavam locais sagrados. Ao associar RO com a religião houve significância estatística com $p=0,00$, demonstrando que dos 23 residentes que frequentavam templos algumas vezes, 11 (47,85\%) se declararam católicos. Da mesma forma, dos 11 residentes que não frequentavam templos todos os 11(100\%) afirmaram não possuir filiação religiosa. Além disso, no contexto de Religiosidade Não Organizacional (RNO), a maioria $21(38,18 \%)$ realizava leituras de livros sagrados, preces ou orações e/ou estudos religiosos diariamente sendo $6(28,57 \%)$ residentes do programa de Residência em Odontologia em Saúde Coletiva e $8(14,55 \%)$ afirmaram rara ou ausência dessa prática sendo a maioria do programa de residência Multiprofissional em Saúde da Família com 3 (37,50\%) residentes. Já nos itens que se referem à Religiosidade Intrínseca (RI), a nota do escore variou de 3 (Menor RI) a 15 (Maior RI), com a média de 6,31 (DP=3,13) (Tabela 3). Houve significância estatística ao associar sexo e RI com $\mathrm{p}=0.0156$, sendo a média maior para o sexo masculino (7.85 com desvio de 3.99 ) que no sexo feminino (5.78 com desvio de 2.64).

Tabela 3. Distribuição da frequência das respostas dadas pelos residentes obtida pela escala Duke Religion Index (DUREL) nas dimensões Religiosidade Organizacional (RO) e Religiosidade Não Organizacional (RNO). Recife, 2020.

\begin{tabular}{lcc}
\multicolumn{2}{|c}{ Questão 1 (RO): Com que frequência você vai a uma igreja, templo ou outro encontro religioso? } \\
& $\begin{array}{c}\text { Frequência } \\
\text { (N=55) }\end{array}$ & (\%) \\
\hline 1. Mais do que uma vez por semana & 08 & 14,55 \\
\hline 2. Uma vez por semana & 06 & 10,91 \\
\hline 3. Duas a três vezes por mês & 04 & 7,27 \\
\hline 4. Algumas vezes por ano & 23 & 41,82 \\
\hline 5. Uma vez por ano ou menos & 03 & 5,45 \\
\hline 6. Nunca & 11 & 20 \\
\hline Questão 2 (RNO): Com que frequência você dedica o seu tempo a atividades religiosas individuais, \\
como preces, rezas, meditaçôes, leitura da bíblia ou de outros textos religiosos?
\end{tabular}


A nota do escore de orientação espiritual, isto é, a relevância e influência da espiritualidade na vida da pessoa aferido pela escala SSRS variou de 8 (mínima) a 30 (máxima), com média de 23,43 (DP=4,87). Ao associar a SSRS com a RO houve significância estatística com $\mathrm{p}=0,00$ destacando-se que os 8 residentes que frequentam templos sagrados mais de uma vez por semana alcançaram média SSRS maior que o geral chegando a 28.75 com desvio padrão de 2.05

\section{Discussão}

O Módulo Transversal de Espiritualidade e Integralidade da SESAU Recife contemplou 7 programas de Residência em Saúde no ano de 2019, sendo esses: Residência em Enfermagem em Atendimento Pré-Hospitalar; Residência em Enfermagem Obstétrica; Residência em Odontologia em Saúde Coletiva; Residência Multiprofissional em Vigilância em Saúde; Residência Multiprofissional na Rede de Atenção Psicossocial; Residência Multiprofissional em Saúde Coletiva; Residência Multiprofissional em Saúde da Família, os programas de Residência em Medicina de Família e Comunidade e Residência em Psiquiatria não foram contemplados nesta edição por incompatibilidade de agendas.

Neste sentido, o Módulo contemplou 62 residentes e destes, 55 participaram do presente estudo, visto que 7 residentes não estavam presentes no momento da coleta de dados.

Destes 55 residentes $74,55 \%$ eram do sexo feminino, a idade média era de $25,41$ anos ( $\mathrm{DP}=4,34), 56,36 \%$ autodeclararam-se da raça branca e 65,45\% afirmaram terem uma filiação religiosa. Estes dados estão de acordo com estudo publicado em periódico brasileiro em 2020, no qual é descrito o perfil dos residentes de programas profissionais em saúde (Uniprofissionais e Multiprofissionais) de universidade pública em Uberlândia. Neste, os residentes eram em sua maioria do sexo feminino $78 \%$, com idade média de 26,68 anos (DP-3,93), sendo $67,70 \%$ brancos e $74,56 \%$ afirmaram ter filiação religiosa ${ }^{16}$. Estes dados corroboram com uma busca de formação qualificada crescente pelo sexo feminino ${ }^{17}$ descritos pela Coordenação de Aperfeiçoamento de Pessoal de Nível Superior (CAPES) em 2016, onde foram identificadas 165.564 mulheres matriculadas e tituladas em cursos de mestrado e doutorado em detrimento de 138.462 homens. O predomínio da raça branca nos espaços de formação ainda é uma realidade nacional, o que pode ser visto no último censo do IBGE de 2010 onde $73,2 \%$ dos pós-graduandos eram desta raça ${ }^{18}$. A filiação religiosa alta igualmente pode ser associada à grande religiosidade apresentada pelos brasileiros onde, segundo o IBGE, em torno de $92 \%$ destes declaram ter uma religião ${ }^{18}$.

Em relação a renda mensal no presente estudo 56,36\% dos residentes declararam renda mensal de 4 a 7 salários-mínimos e $65,45 \%$ possuíam a graduação como maior título. Em comparação com o estudo de Uberlândia onde é descrito que $54 \%$ dos residentes declararam renda familiar entre 2 e 3 salários-mínimos e 89,83\% dos residentes possuíam graduação como maior título. Esses dados podem ser justificados pois no caso de Uberlândia $93,27 \%$ dos residentes iniciaram a residência até um ano após a conclusão na graduação e 64,40\% destes não tinham experiencia profissional tendo por isso pouco tempo para cursar outras pós-graduações como também ter uma renda mais elevada ${ }^{16}$. No Recife $34,55 \%$ dos residentes tem outra especialização e ou mestrado/doutorado indicando maior tempo entre a graduação e o ingresso no programa atual, desta forma permitindo atuação profissional remunerada.

No presente trabalho $94,55 \%$ dos residentes declararam acreditar que a espiritualidade interfere muito ou extremamente na saúde sendo que $92,72 \%$ destes consideram essa interferência geralmente positiva ou igualmente positiva e negativa. Para 90,91\% dos residentes a abordagem do tema é muito ou muitíssimo pertinente. Estes dados estão em acordo com estudo de 2019, que avaliou opiniões de tutores 
de medicina acerca da temática de espiritualidade em uma faculdade no nordeste brasileiro. Neste, $88,37 \%$ de 86 tutores afirmaram que a espiritualidade interfere muito ou extremamente na saúde sendo que $89,5 \%$ afirmaram que esta influência é positiva ou geralmente positiva e ainda $62,79 \%$ consideravam a abordagem da temática muito ou muitíssimo pertinente ${ }^{19}$. Estes dados são importantes pois, à medida em que são descobertas formas de melhorar o cuidado na assistência à saúde, estas devem ser implantadas de imediato, visto ser dever do profissional de saúde ofertar o que há de melhor, baseado em evidências, objetivando o cuidado integral dos pacientes.

Neste mesmo estudo ${ }^{19}, 72,94 \%$ dos tutores afirmaram que algumas vezes ou frequentemente estiveram motivados para abordar a espiritualidade do paciente e apenas 10,47\% consideraram-se como muito preparados para tal. O estudo ainda descreve que o desencorajamento em abordar o tema na prática clínica ocorria em $62,79 \%$ tutores, por falta de treinamento prático ou conhecimento teórico e em $24,42 \%$ destes, por falta de tempo e medo de impor pontos de vista religiosos ao paciente ${ }^{19}$. Nestes aspectos existe concordância com o presente estudo uma vez que no exercício prático durante a formação na residência 77,77\% dos residentes afirmaram que estiveram motivados frequentemente ou algumas vezes, apesar de apenas $21,82 \%$ consideraram estar muito preparados para abordar o tema com os pacientes, sendo que a falta de coragem na abordagem do tema deu-se em $61,81 \%$ destes devido à falta de conhecimento teórico ou treinamento prático e em $36,36 \%$ por medo de impor os próprios pontos de vista religiosos ao paciente. Estes dados vêm reforçar a necessidade de uma maior qualificação sobre espiritualidade nos processos formativos em saude, desde a graduação, pós-graduações e até processsos de atualização e aperfeiçoamento de conteúdos e práticas na prática clinica.

Ainda relacionado à prática durante a formação, 87,04\% afirmaram que, ao longo da residência, os docentes/tutores/preceptores nunca ou raramente abordaram temas referentes à espiritualidade e $83,64 \%$ deles afirmaram que abordaram raramente ou algumas vezes temas referentes à espiritualidade nos campos de prática. Da mesma forma, em estudo que avaliou um módulo transversal de ensino na graduação de medicina sobre espiritualidade em faculdade no estado de Pernambuco, os autores identificaram que a maior parte $(51,5 \%)$ dos 305 estudantes que participaram da pesquisa relataram que essa abordagem raramente ou nunca esteve presente ${ }^{10}$. Esta diferença de frequência de abordagem da espiritualidade sendo maior na graduação que na pós graduação pode estar relacionada às novas Diretrizes Curriculares Nacionais para os cursos de Medicina, que definem que a abordagem dos conteúdos devem conter assuntos que envolvam a saúde e o ser humano no seu contexto integral, isto é biológico, emocional, mente, social e espiritual ${ }^{20}$. Em relação a abordagem na prática profissional em espiritualidade, estudo realizado na Faculdade Pernambucana de Saúde em Recife (PE), onde foram avaliados 71 tutores dos cursos de Enfermagem, Farmácia, Fisioterapia, Nutrição e Psicologia, 73,24\% afirmaram que abordaram raramente ou algumas vezes a temática com os pacientes ${ }^{21}$.

Nos quesitos conteúdo e ensino-aprendizagem, os residentes julgaram o Módulo Transversal de Espiritualidade e Integralidade da seguinte forma: Em geral, para $94,55 \%$ o módulo era muito bom e para $5,45 \%$ bom; dos conteúdos, $80 \%$ acharam mais interessante Espiritualidade e Finitude seguido de Espiritualidade e Humanização e Espiritualidade na Prática Clínica, estes dois últimos com $76,36 \%$ cada. Estudo que igualmente abordou um módulo transversal em espiritualidade, com características semelhantes ao realizado no presente estudo, porém realizado com estudantes de graduação de medicina, identificou o seguinte: Para 46,6\% dos estudantes o módulo era muito bom e para 38,0\% bom; Dos conteúdos, Espiritualidade na Prática Clínica e Espiritualidade e Humanização foram preferidos por igual percentual de discentes (51,50\%), seguidos por Psiconeuroimunologia e Epigenética, ambos apontados por $36,70 \%$ dos discentes ${ }^{10}$. Os autores deste trabalho acreditam que este resultado positivo possa estar relacionado ao fato de ambos os módulos terem seus conteúdos 
embasados nas National Competencies in Spirituality and Health for Medican Education (NCSMD) elaboradas pela Association of American Medical Colleges (AAMC) ${ }^{11,12}$, assim como utilizarem de metodologias ativas de ensino e aprendizagem, que estimulam o raciocínio crítico e reflexivo envolvendo o estudante como protagonista do seu aprendizado ${ }^{22}$.

Todos os participantes afirmaram que o módulo contribuiu em suas vidas, seja profissional para $96,36 \%$ dos residentes e pessoal para $98,18 \%$ destes. Além disso, a quase totalidade dos residentes $92,73 \%$ afirmaram sentir-se mais preparado para abordar a espiritualidade na prática clínica como profissional de saúde após a participação no módulo. No estudo previamente citado, realizado com estudantes de medicina, 96,30\% afirmaram que o módulo contribuiu para sua prática profissional futura e ainda $91,80 \%$ disseram sentirem-se mais praparados para abordar a espiritualidade ${ }^{10}$. Apesar da contribuição positiva que a formação em espiritualidade pode trazer as profissões de saúde, a literatura aponta que o Brasil, apesar de ser um grande produtor de pesquisas científicas no tema, o que pode ser visto no crescimento de $280 \%$ nestas produções entre 2004 e 2014 , apenas $16 \%$ das faculdades de graduação em psicologia abordavam a temática, ao menos 34,9\% de docentes de enfermagem afirmaram que nas faculdades onde têm seus vínculos a temática não é abordada ${ }^{23}$, sendo pior este cenário ao tratar de pós-graduação ${ }^{24}$.

A respeito da continuidade e ampliação da oferta do módulo de espiritualidade e integralidade, $98,18 \%$ dos residentes afirmaram que ele deve ser ofertado aos futuros residentes e todos afirmaram que este deve ser ofertado para cursos de pós-graduação em outras instituições. Da mesma forma, dos estudantes de medicina questionados sobre estas ofertas de formação em espiritualidade na graduação, 91,80\% afirmaram que o curso deve continuar a ser ofertado no curso de medicina e $99 \%$ indicaram que deve ser ampliado para os outros cursos de graduação ${ }^{10}$. Os autores do presente estudo entendem que estes resultados possam estar ligados aos aprendizados que a formação em espiritualidade possa trazer, não somente nos domínios cognitivos e psicomotores mas principalmente no domínio das atitudes, trazendo reflexões importantes em bases como saber conviver e saber ser ${ }^{25}$.

Sobre as concepções acerca da espiritualidade e religiosidade, avaliada pela escala DUREL, a média observada na dimensão da RI pelo presente estudo $(6,31 \pm 3,13)$ encontra-se consideravelmente inferior ao encontrado em tutores de medicina $(11,74 \pm 3,46)^{19}$ da mesma forma que em estudantes de medicina $(10,78 \pm 3,40)^{10}$. Estes dados podem indicar o desafio que é o desenvolvimento da consciência espiritual durante a formação profissional na pós graduação $0^{26}$, talvez por ser um momento de grande descoberta e aprofundamento em saberes técnicos. Ao tratar da $\mathrm{RO}$ estudo realizado com estudantes de graduação em medicina ${ }^{10}$ demostrou que $31,50 \%$ dos estudantes frequentavam instituições religiosas algumas vezes por ano e $19,30 \%$ destes nunca frequentavam. Achado este em acordo com o presente estudo que demostrou que $41,82 \%$ dos residentes frequentam instituições religiosas algumas vezes e $20 \%$ não frequentam. Importante destacar que frequentar ou não instituições religiosas não indica necessariamente uma religiosidade elevada, apesar de indicar uma maior possibilidade de formação de uma rede de apoio para auxílio em necessidades como algum problema de saúde ${ }^{1}$. Neste sentido os dados aqui descritos são indicativos que estes residentes podem usufruir de uma rede de apoio em caso de necessidades. Em relação a RNO este mesmo estudo ${ }^{10}$ descreveu que 37\% dos estudantes dedicamse diariamente a atividades religiosas pessoais ou individuais, como preces, orações e/ ou meditações com fins religiosos individuais concordando com o presente estudo que aferiu estas características em 38,18\% dos residentes. Estes dados sugerem que os residentes demonstram uma vivência subjetiva de sua espiritualidade/religiosidade, o que em última instância favorece hábitos de vida saudáveis e melhor saúde mental ${ }^{4}$.

Em relação a escala SSRS o total foi de $23,43(\mathrm{DP}=4,87)$, semelhante ao encontrado em estudo realizado em tutores da área de saúde $(23,21 \pm 5,94)^{19}$, como também 
aos resultados obtidos entre estudantes de medicina $(21,29 \pm 5,54)^{10}$ resultados considerados elevados segundo o estudo original de validação da escala ${ }^{27}$. Importante salientar que os itens avaliados na escala refletem a orientação espiritual do indivíduo, ou seja, se ele considera ou julga importante (mais ou menos) questões pertinentes a sua dimensão espiritual e as aplica em sua vida ${ }^{27}$. Neste sentido, pode-se inferir que os residentes do presente estudo julgam importantes as questões espirituais em suas vidas.

\section{Conclusões}

A espiritualidade/religiosidade dos residentes aferidas mostraram níveis considerados altos de orientação espiritual e na sua maioria hábitos importantes ralcionados as RO e RNO, porém níveis de RI considerados baixos ao comparar com os resultados obtidos em outros estudos.

Estes achados podem indicar que os residentes dos programas de residencia estudados possuem alguns hábitos importantes relacionados a espiritualidade/ religiosidade como frequentação de instituições religiosas, realização de orações / preces, crença em um ser superior e utilização destas crenças como guias em suas vidas porém possuem dificuldade em ter estes valores de forma internalizada sem necessidade de práticas exteriores.

O Módulo foi positivamente avaliado pela maioria dos residentes em praticamente a totalidade dos questionamentos. Estes abordavam desde a motivação na participação nas aulas, relevância do módulo, conteúdo teórico e prático, contribuição pessoal, profissional e social promovido e ainda a sensação de preparo para abordar a espiritualidade na prática clínica como profissional de saúde após a participação no módulo.

Além disso, os residentes, na sua maioria, informaram que o ensino em espiritualidade é bastante importante e deve ser incluído na matriz curricular de forma permanente dos programas de residência em saúde, mantendo a oferta aos residentes da SESAU e ampliando-a para programas de residência de outras instituições.

Evidenciou-se que, para o grupo estudado, a formação em espiritualidade é relevante e traz contribuições positivas à prática profissional dos residentes, sendo recomendado que esta formação seja institucionalizada e ampliada. São necessários, no entanto, mais estudos para avaliar outras propostas semelhantes, proporcionando uma visão mais abrangente da temática, considerando o envolvimento de outros cenários assistenciais, categorias (ex.: residências médicas) e metodologias de ensino-aprendizagem

\section{Referências}

1. Koenig HG, McCullough ME, Larson DB. Handbook of religion and health [Internet]. Questia; 2001 [citado em 2018 Mar 18]. Disponível em: https://www.questia.com/read/106205934/handbook-of-religion-and-health

2. Puchalski C, Ferrell B, Virani R, et al. Improving the quality of spiritual care as a dimension of palliative care: the report of the consensus conference. J Palliat Med. 2009;12(10):885-904. http://dx.doi.org/10.1089/jpm.2009.0142. PMid:19807235.

3. Lucchetti G, Lucchetti ALG. Spirituality, religion, and health: over the last 15 years of field research (1999-2013). Int J Psychiatry Med. 2014;48(3):199-215. http://dx.doi.org/10.2190/PM.48.3.e. PMid:25492714.

4. Koenig HG. Religion, spirituality, and health: the research and clinical implications. ISRN Psychiatry. 2012;2012:278730. http://dx.doi.org/10.5402/2012/278730. PMid:23762764.

5. Lucchese F, Koenig H. Religion, spirituality and cardiovascular disease: research, clinical implications, and opportunities in Brazil. Rev Bras Cir Cardiovasc. 2013;28(1):103-28. http://dx.doi.org/10.5935/1678-9741.20130015. PMid:23739939.

6. Gobatto CA, Araujo TCCF. Religiosidade e espiritualidade em oncologia: concepções de profissionais da saúde. Psicol USP. 2013;24(1):11-34. http://dx.doi.org/10.1590/S0103-65642013000100002.

7. Bonnie B, Puchalski CM, Washington G. More schools teaching spirituality in medicine [Internet]. 2008 [citado em 2018 Mar 18]. Disponível em: http://dental.buffalo.edu/content/dam/www/news/imported/pdf/March08/AMNHolmesSpirituality. pdf 
8. Lucchetti G, Lucchetti ALG, Espinha DCM, de Oliveira LR, Leite JR, Koenig HG. Spirituality and health in the curricula of medical schools in Brazil. BMC Med Educ. 2012;12(1):78. http://dx.doi.org/10.1186/1472-6920-12-78. PMid:22900476.

9. Fortin AH 6th, Barnett KG. Medical school curricula in spirituality and medicine. JAMA. 2004;291(23):2883. http://dx.doi. org/10.1001/jama.291.23.2883. PMid:15199044.

10. Ferreira AGC, Oliveira JAC, Jordán APW. Educação em saúde e espiritualidade: uma proposta de transversalidade na perspectiva do estudante. Interdiscip J Heal Educ. 2016;1(1):3-12. http://dx.doi.org/10.4322/ijhe2016005.

11. Association of American Medical Colleges. Medical School objectives project: report III Contemporary issues in medicine: communication in medicine [Internet]. 1999 [citado em 2021 Mar 28]. Disponível em: http://timetravel.mementoweb.org/ memento/1999/http:/www.aamc.org/meded/msop/

12. Puchalski CM, Blatt B, Kogan M, Butler A. Spirituality and health: the development of a field. Acad Med. 2014;89(1):10-6. http://dx.doi.org/10.1097/ACM.0000000000000083. PMid:24280839.

13. Lucchetti G, De Oliveira LR, Koenig HG, Leite JR, Lucchetti AL. Medical students, spirituality and religiosity-results from the multicenter study SBRAME. BMC Med Educ. 2013;13(1):162. http://dx.doi.org/10.1186/1472-6920-13-162. PMid:24314327.

14. Moreira-Almeida A, Peres MF, Aloe F, Lotufo F No. Versão em português da Escala de Religiosidade da Duke - DUREL. Rev Psiquiatr Clin. 2008;35(1):31-2. http://dx.doi.org/10.1590/S0101-60832008000100006.

15. Gonçalves AMS, Pillon SC. Adaptação transcultural e avaliação da consistência interna da versão em português da Spirituality Self Rating Scale (SSRS). Rev Psiquiatr Clin. 2009;36(1):10-5. http://dx.doi.org/10.1590/S0101-60832009000100002.

16. Oliveira SV, Camargos SPS. Perfil, qualidade de vida e perspectivas futuras de residentes do programa de residência em área profissional da saúde. Rev Educ em Saúde [Internet]. 2020 [citado em 2021 Mar 28];8(1):50-63. Disponível em: http:// periodicos.unievangelica.edu.br/index.php/educacaoemsaude/article/view/4138/3284

17. Santos AO. Mulheres permanecem como maioria na pós-graduação brasileira [Internet]. Portal de Periódicos da Capes; 2018 [citado em 2021 Dez 23]. Disponível em: https://www.gov.br/capes/pt-br/assuntos/noticias/ mulheres-permanecem-como-maioria-na-pos-graduacao-brasileira

18. Instituto Brasileiro de Geografia e Estatística. IBGE Censo 2010: resultados [Internet]. 2016 [citado em 2020 Dez 23 ]. Disponível em: https://censo2010.ibge.gov.br/resultados.html

19. Campos ITM, Bastos BLS, Santos ISM, Araújo PFB, Jordán APW. Educação em saúde e espiritualidade na perspectiva de tutores de medicina da Faculdade Pernambucana de Saúde (FPS). Interdisciplinary Journal of Health Education. 2020;5. https://doi.org/10.4322/ijhe.2020.011.

20. Ferreira MJM, Ribeiro KG, Almeida MM, et al. New national curricular guidelines of medical courses: opportunities to resignify education. Interface Commun Heal Educ. 2019;23(Suppl. 1):e170920. http://dx.doi.org/10.1590/interface.170920.

21. Ferreira MC, Vasconcelos AG, Freitas LMS, Jordán APW, Medeiros FPM, Viana OS. Educação em saúde e espiritualidade na percepção de docentes em saúde de uma instituição de ensino superior do Recife-Brasil [trabalho de conclusão]. Recife: Faculdade Pernambucana de Saúde; 2020 [citado em 2020 Dez 23]. Disponível em: http://tcc.fps.edu.br:80/jspui/handle/ fpsrepo/933

22. Silva MKD, Acosta BS, Silva EB, Souza NS, Beck CLC, Silva KKD. Active learning methodologies: possible paths to innovation in health teaching. Esc Anna Nery. 2018;22. http://dx.doi.org/10.1590/2177-9465-ean-2017-0435.

23. Damiano RF, Lucchetti ALG, Lucchetti G. Ensino de "saúde e espiritualidade" na graduação em medicina e outros cursos da área de saúde. HU Rev. 2020;44(4):515-25. http://dx.doi.org/10.34019/1982-8047.2018.v44.25928.

24. Jordán APW, Barbosa LNF. Espiritualidade e formação nos programas de residência em saúde de uma cidade no nordeste brasileiro. Rev Bras Educ Med. 2019;43(3):82-90. http://dx.doi.org/10.1590/1981-52712015v43n3rb20180221.

25. Borges FAF. Educação do Indivíduo para o século XXI: o relatório Delors como representação da perspectiva da Unesco. Rev Labor. 2017;1(16):12. http://dx.doi.org/10.29148/labor.v1i16.6504.

26. Banin LB, Suzart NB, Banin VB, Guimarães FG, Mariotti LL, Lucchetti G. Spirituality: do teachers and students hold the same opinion? Clin Teach. 2013;10(1):3-8. http://dx.doi.org/10.1111/j.1743-498X.2012.00576.x. PMid:23294736.

27. Galanter M, Dermatis H, Bunt G, Williams C, Trujillo M, Steinke P. Assessment of spirituality and its relevance to addiction treatment. J Subst Abuse Treat. 2007;33(3):257-64. http://dx.doi.org/10.1016/j.jsat.2006.06.014. PMid:17574800.

\section{Contribuição dos autores}

Arturo de Pádua Walfrido Jordán contribuiu na concepção e desenvolvimento do estudo, desenho metodológico, supervisão, coleta e tratamento dos dados, análise/ interpretação, levantamento da literatura, redação e revisão crítica. Daniella Bandim Cruz contribuiu na coleta e tratamento dos dados tratamento dos dados, análise/ interpretação, levantamento da literatura, redação e revisão crítica. Arthur Fernandes da Silva análise/interpretação, levantamento da literatura, redação e revisão crítica. Leopoldo Nelson Fernandes Barbosa análise/interpretação, levantamento da literatura, redação e revisão crítica. 


\section{Anexo A. Instrumento de coleta de dados.}

AVALIAÇÃO DO MÓDULO TRANSVERSAL DE ESPIRITUALIDADE E INTEGRALIDADE OFERTADO AOS PROGRAMAS DE RESIDÊNCIA EM SAÚDE DA SECRETARIA DE SAÚDE DO RECIFE.

Você está sendo convidado a participar dessa pesquisa que tem o objetivo de analisar a sua opinião e entendimento, enquanto residente dos programas de residencias em saude da secretaria de saude do Recife, com duração media de 15 minutos, acerca da temática espiritualidade em saúde e sua aplicabilidade na formação e prática profissional.

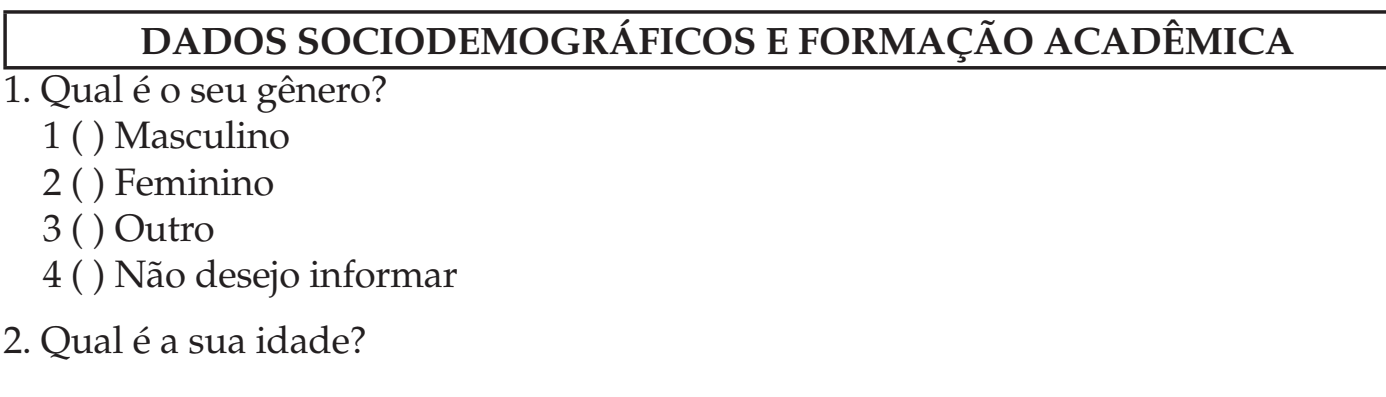

3. Em relação a sua etnia, você se considera:
1 ( ) Branco
2 ( ) Negro
3 ( ) Oriental
4 ( ) Outra

4. Qual é a renda da sua família?

1 ( ) 1 a 3 salários mínimos

2 ( ) 4 a 7 salários mínimos

3 ( ) 8 a 12 salários mínimos

4 ( ) Mais de 12 salários mínimos

5. Qual é sua maior formação acadêmica?
1 ( ) Graduação
2 ( ) Especialização / Residencia em Saúde
3 ( ) Mestrado
4 ( ) Doutorado
5 ( ) Pós doutorado

6. Qual é o programa de residência que você está cursando?
( ) Residência em Enfermagem em Atendimento Pré-Hospitalar
( ) Residência de Medicina de Família e Comunidade
( ) Residência Médica em Psiquiatria
( ) Residência em Enfermagem Obstétrica
( ) Residência em Odontologia em Saúde Coletiva
( ) Residência Multiprofissional em Vigilância em Saúde
( ) Residência Multiprofissional na Rede de Atenção Psicossocial
( ) Residência Multiprofissional em Saúde Coletiva
( ) Residência Multiprofissional em Saúde da Família

DADOS ACERCA DA TEMÁTICA “SAÚDE E ESPIRITUALIDADE"

O que você entende por "Espiritualidade"? Caso julgue pertinente, assinale mais de uma resposta.

1 ( ) Busca de sentido e significado para a vida humana, apenas

2 ( ) É uma busca pessoal pelo entendimento de respostas a questões sobre a vida, seu significado e relações com o sagrado e transcendente, que pode ou não estar relacionada a propostas de uma determinada religião 
3 ( ) Crença em Deus e/ou na vida após a morte

4 ( ) Envolve o entendimento das questões últimas da vida, com o surgimento provável de rituais e congregações religiosas

5 ( ) Dimensão de cada ser humano, subjetiva e pessoal

8. Você relaciona a temática "Saúde e Espiritualidade" com: Caso julgue pertinente, assinale mais de uma resposta.

1 ( ) Integralidade, Saúde Única e Holismo em saúde

2 ( ) Humanização da prática médica

3( ) Qualidade de vida

4 ( ) Interferência positiva ou negativa na saúde

5 ( ) Nenhuma das alternativas

6 ( ) Outro

9. No geral, o quanto você acha que a Espiritualidade interfere na saúde das pessoas?

1 ( ) Muito pouco

2 ( ) Pouco

3 ( ) Mais ou menos

4 ( ) Muito

5 ( ) Extremamente

10. No geral, a influência que a Espiritualidade exerce na saúde das pessoas é:

1 ( ) Positiva

2 ( ) Geralmente positiva

3 ( ) Igualmente positiva e negativa

4 ( ) Geralmente negativa

5 ( ) Negativa

11. Você sente vontade de abordar a Espiritualidade dos pacientes?

1 ( ) Nunca estive motivado

2 ( ) Raramente estive motivado

3 ( ) Algumas vezes estive motivado

4 ( ) Frequentemente estive motivado

5 ( ) Sempre estive motivado

12. O quanto você se considera preparado para abordar a espiritualidade dos pacientes?

1 ( ) Pouquíssimo preparado

2 ( ) Pouco preparado

3 ( ) Moderadamente preparado

4 ( ) Muito preparado

5 ( ) Muitíssimo preparado

13. O quanto você considera pertinente tal abordagem?

1 ( ) Pouquíssimo pertinente

2 ( ) Pouco pertinente

3 ( ) Moderadamente pertinente

4 ( ) Muito pertinente

5 ( ) Muitíssimo pertinente

14. Caso você se sinta desencorajado a abordar a espiritualidade dos pacientes, assinale a afirmação que melhor se enquadra. Caso julgue pertinente, assinale mais de uma resposta.

1 ( ) Falta de conhecimento teórico

2 ( ) Falta de treinamento prático

3 ( ) Falta de tempo 
4 ( ) Desconforto com o tema

5 ( ) Medo de impor pontos de vista religiosos ao paciente

6 ( ) Não faz parte do meu ofício enquanto profissional da saúde

7 ( ) Outro

DADOS ACERCA DO ENSINO EM SAÚDE E ESPIRITUALIDADE

15. Ao longo do seu período de residência, o quanto você abordou temas referentes a espiritualidade nos campos de prática nas atividades curriculares do programa:

1 ( ) Nunca

2 ( ) Raramente

3 ( ) Algumas vezes

4 ( ) Frequentemente

5 ( ) Sempre

16. Ao longo da sua formação acadêmica, os docentes/tutores/preceptores abordaram temas referentes a espiritualidade nas atividades curriculares?

( ) Nunca

( ) Raramente

( ) Algumas vezes

( ) Frequentemente ( ) Sempre

17. O residente da área da saúde deve ser preparado, durante sua formação, para abordar a Espiritualidade dos pacientes?

1 ( ) Não

2() Um pouco

3 ( ) Razoavelmente

4 ( ) Muito

5 () Bastante

6 ( ) Não tenho opinião formada

18. O quanto você acha importante incluir o ensino em Saúde e Espiritualidade como matriz curricular nos Programas de Residencia:

1 ( ) Não acho importante

2 ( ) Um pouco

3 ( ) Razoavelmente

4 ( ) Muito

5 ( ) Bastante

6 ( ) Não tenho opinião formada

DADOS ACERCA DA APLICABILIDADE CLÍNICA EM SAÚDE E ESPIRITUALIDADE

19. Em relação a sua última consulta (ou suas últimas 3 consultas), você: Caso julgue pertinente, assinale mais de uma resposta.

1 ( ) Perguntou se o paciente possui uma fé ou crença, institucionalizada ou não

2 ( ) Perguntou a atual situação do paciente em relação a sua fé, se este relatar presença

3 ( ) Perguntou se o paciente acredita que sua fé irá aliviar, ajudar ou influenciar em seu tratamento

4 ( ) Estimulou as práticas religiosas e/ou espirituais de seu paciente

5 ( ) Não abordou a religiosidade e/ou espiritualidade do paciente

20. Com que frequência você leva em consideração a espiritualidade/religiosidade trazida pelo seu paciente durante a consulta?

1 ( ) Nunca

2 ( ) Raramente 
3 ( ) Algumas vezes

4 ( ) Frequentemente

5 ( ) Sempre

21. Se já conseguiu abordar a espiritualidade em uma consulta, em que situação isso ocorreu:

1 ( ) Questionou diretamente sobre a espiritualidade do paciente

2 ( ) Usou a espiritualidade para fazer um paciente aderir ao tratamento

3 ( ) Usou a espiritualidade para acalmar o paciente em uma situação difícil

4 ( ) Quando foi necessário por uma urgência clinica

5 ( ) Não abordou em nenhuma situação

22. Ao longo de sua prática clínica, com que frequência você abordou conceitos sobre "Saúde e Espiritualidade" durante a consulta:

1 ( ) Nunca

2 ( ) Raramente

3 ( ) Algumas vezes

4 ( ) Frequentemente

5 ( ) Sempre

23. Qual a alternativa que melhor descreve a sua filiação religiosa:

1 ( ) Não possuo filiação religiosa

2 ( ) Evangélico/Protestante

3 ( ) Católico

4 ( ) Espírita

5 ( ) Outra

\section{DADOS ACERCA DO MÓDULO TRANSVERSAL EM ESPIRITUALIDADE E INTEGRALIDADE}

24. Qual o seu conceito em relação ao Módulo Transversal em Espiritualidade e Integralidade?
( ) Muito fraco
( ) Fraco
( ) Regular
( ) Bom
( ) Muito bom

25. O quanto você considera pertinente tal abordagem?
( ) Pouquíssimo pertinente
( ) Pouco pertinente
( ) Moderadamente pertinente
( ) Muito pertinente
( ) Muitíssimo pertinente

26. Você acredita que a participação no Módulo Transversal em Espiritualidade e Integralidade lhe trouxe contribuições?

( ) Sim

( ) Não (pule para a questão 29)

27. Em qual instancia você enquadraria a(s) contribuição(ções) do Módulo Transversal em Espiritualidade e Integralidade? Caso julgue pertinente, assinale mais de uma resposta.

( ) Contribuições para minha prática profissional

( ) Contribuições para minha vida pessoal

( ) Contribuições para minha vida em sociedade

28. Você acredita que esta contribuição poderá ser aplicada na sua prática profissional? 

( ) Sim
( ) Não
( ) Não tenho opinião formada

29. Você acredita que a participação no Módulo Transversal em Espiritualidade e Integralidade contribuiu com suas crenças e condutas em relação à espiritualidade e a religiosidade?

( )Sim

( ) Não

( ) Não tenho opinião formada

30. Qual a temática que foi abordada no Módulo Transversal em Espiritualidade e Integralidade você achou mais interessante? Caso julgue pertinente, assinale mais de uma resposta.

( ) Conceitos básicos sobre espiritualidade, religiosidade e religião

( ) Psiconeuroimunologia

( ) Epigenética

( ) Espiritualidade e Humanização

( ) Espiritualidade e Finitude

( ) Espiritualidade na Prática Clínica

31. Quanto a sua motivação em frequentar as aulas do Módulo Transversal em Espiritualidade e Integralidade:

( ) Nunca estive motivado

( ) Raramente estive motivado

( ) Algumas vezes estive motivado

( ) Frequentemente estive motivado

( ) Sempre estive motivado

32. Após a participação no Módulo Transversal em Espiritualidade e Integralidade você se sente mais preparado para abordar a espiritualidade na sua prática clínica como profissional de saúde?

( ) Sim

( ) Não

( ) Não tenho opinião formada

33. Você acredita que a Secretaria de Saúde de Recife deveria oferecer aos residentes, no futuro, novamente o Módulo Transversal em Espiritualidade e Integralidade?

( ) Sim

( ) Não

( ) Não tenho opinião formada

34. Você acredita que o conteúdo abordado no Módulo Transversal em Espiritualidade e Integralidade interessa também a residentes de outros programas inclusive de outras instituições?

( ) Sim

( ) Não

( ) Não tenho opinião formada

35. Excetuando o Módulo Transversal em Espiritualidade e Integralidade, você já participou de alguma outra atividade de formação sobre a temática "Saúde e Espiritualidade"?

( ) Sim (grupos de estudo, ligas acadêmicas etc)

( ) Não

DADOS ACERCA DE CONCEPÇÕES DE ESPIRITUALIDADE E RELIGIOSIDADE

36. Duke Religion Index- DUREL 
36 (A) Com que frequência você vai a uma igreja, templo ou encontro religioso?

1 ( ) Mais de uma vez por semana

2 ( ) Uma vez por semana

3 ( ) Duas a três vezes por mês

4 ( ) Algumas vezes por ano

5 ( ) Uma vez por ano

6 ( ) Nunca

36 (B) Com que frequência você dedica seu tempo a atividades religiosas, pessoais ou individuais, como preces, orações, meditações, leitura de livros sagrados ou de outros textos religiosos?

1 ( ) Mais de uma vez ao dia

2 ( ) Diariamente

3 ( ) Duas ou três vezes por semana

4 ( ) Uma vez por semana

5 ( ) Poucas vezes por mês

6 ( ) Raramente ou nunca

36 (C) Em minha vida, eu sinto a presença de Deus (ou do Espírito):

1 ( ) Totalmente verdade pra mim

2 ( ) Em geral é verdade

3 ( ) Não estou certo

4 ( ) Em geral não é verdade

5 ( ) Não é verdade

36 (D) Minhas crenças religiosas estão realmente por trás de toda a minha maneira de viver:

1 ( ) Totalmente verdade pra mim

2 ( ) Em geral é verdade

3 ( ) Não estou certo

4 ( ) Em geral não é verdade

5 ( ) Não é verdade

36 (E) Eu me esforço para viver minha religião em todos os aspectos da minha vida:

1 ( ) Totalmente verdade pra mim

2 ( ) Em geral é verdade

3 ( ) Não estou certo

4 ( ) Em geral não é verdade

5 ( ) Não é verdade

37. Spirituality Self Rating Scale - SSRS

\begin{tabular}{|c|c|c|c|c|c|}
\hline & $\begin{array}{c}\text { COSCORDO } \\
\text { MUITO }\end{array}$ & CONCORDO & $\begin{array}{c}\text { CONCORDO } \\
\text { PARCLALAENTE }\end{array}$ & DISCORDO & $\begin{array}{l}\text { DISCORDO } \\
\text { TOTALMENTE }\end{array}$ \\
\hline $\begin{array}{l}\text { E importante, para mim, passar tempo com } \\
\text { pensamentos espirituais particulares e meditações }\end{array}$ & & & & & \\
\hline $\begin{array}{l}\text { Esforço-me muito para viver minha vida de } \\
\text { acordo com minhas crenças religiosas }\end{array}$ & & & & & \\
\hline $\begin{array}{l}\text { As orações ou pensamentos espirituais que tenho } \\
\text { quando estou sozinho são tão importantes para } \\
\text { mim quanto os teria durante cerimonias religiosas } \\
\text { ou reuniões espirituais }\end{array}$ & & & & & \\
\hline $\begin{array}{l}\text { Eu gosto de ler sobre minha espiritualidade e/ou } \\
\text { minha religião }\end{array}$ & & & & & \\
\hline $\begin{array}{l}\text { A espiritualidade ajuda a manter minha vida } \\
\text { estável e equilibrada, da mesma forma que a } \\
\text { minha cidadania, amizades e sociedade o fazem }\end{array}$ & & & & & \\
\hline $\begin{array}{l}\text { Minha vida toda é baseada na minha } \\
\text { espiritualidade }\end{array}$ & & & & & \\
\hline
\end{tabular}

